\title{
Fixed point theorems for generalized contraction mappings on b-rectangular metric spaces
}

\author{
Cristian Daniel Alecsa
}

\begin{abstract}
In the present article, we study some fixed point theorems for a hybrid class of generalized contractive operators in the context of b-rectangular metric spaces. Examples justifying theorems and an open problem regarding to further generalizations for this type of operators are also given.
\end{abstract}

Mathematics Subject Classification (2010): 47H10, 54H25.

Keywords: Generalized contraction, b-rectangular metric space, expansive mappings, fixed point.

\section{Introduction and preliminaries}

In this section we shall present some useful lemmas and definitions regarding rectangular and b-rectangular metric spaces. Also, we shall present some recent results in the field of fixed point theory concerning expansive operators and some generalized contraction mappings.

In [6], A. Branciari introduced a new metric-type space, when triangle inequality is replaced by an inequality which involves four different elements. This is called a rectangular metric space or a generalized metric space (g.m.s.)

Definition 1.1. Let $X \neq \emptyset, d: X \times X \rightarrow[0, \infty)$, such that for each $x, y \in X$ and $u, v \in X$ (each distinct from $x$ and $y$ ), we have that

(1) $d(x, y)=0 \Longleftrightarrow x=y$,

(2) $d(x, y)=d(y, x)$,

(3) $d(x, y) \leq d(x, u)+d(u, v)+d(v, y)$.

Furthermore, from [10] we mention that convergent sequences and Cauchy sequences can be introduced in a similar manner as in metric spaces.

Also, from the same paper, we know that if $(X, d)$ is a rectangular metric space and if $\left(x_{n}\right)$ is a b-rectangular Cauchy sequence with the property that $x_{n} \neq x_{m}$, for each $n \neq m$, then $\left(x_{n}\right)$ converge to at most one point, i.e. the property that $(X, d)$ is 
Haussdorf becomes superfluous.

Moreover, from [8], [9], [22], we recall the definition of b-rectangular metric spaces (or b-generalized metric spaces), briefly b-g.m.s.

Definition 1.2. Let $X \neq \emptyset, s \geq 1$ be a given real number and $d: X \times X \rightarrow[0, \infty)$, such that for each $x, y \in X$ and $u, v \in X$ (each distinct from $x$ and $y$ ), we have that

(1) $d(x, y)=0 \Longleftrightarrow x=y$,

(2) $d(x, y)=d(y, x)$,

(3) $d(x, y) \leq s[d(x, u)+d(u, v)+d(v, y)]$.

As in metric spaces, we recall the basic notions regarding sequences in b-g.m.s:

Definition 1.3. Let $(X, d)$ be a b-g.m.s, $x \in X$ and $\left(x_{n}\right) \subset X$ be a given sequence. Then

(a) $\left(x_{n}\right)$ is convergent in $(X, d)$ to an element $x \in X$, if for each $\varepsilon>0$, there exists $n_{0} \in \mathbb{N}$, such that $d\left(x_{n}, x\right)<\varepsilon$, for each $n>n_{0}$. We denote this by $\lim _{n \rightarrow \infty} x_{n}=x$.

(b) $\left(x_{n}\right)$ is Cauchy in $(X, d)$ (or b-rectangular Cauchy, briefly b-g.m.s.), if for each $\varepsilon>0$, there exists $n_{0} \in \mathbb{N}$, such that $d\left(x_{n}, x_{n+p}\right)<\varepsilon$, for each $n>n_{0}$ and for each $p>0$. We denote this by $\lim _{n \rightarrow \infty} d\left(x_{n}, x_{n+p}\right)=0$, for each $p>0$.

(c) $(X, d)$ is said to be complete b-g.m.s, if every Cauchy sequence in $\mathrm{X}$ converges to some $x \in X$.

We recall the following important remark from [8]:

Remark 1.4. (1) Every metric space and every rectangular metric space (g.m.s) is b-g.m.s.

(2) The limit of a sequence in a b-rectangular metric space is not unique.

(3) Every convergent sequence in a b-g.m.s is not necessarily a b-g.m.s Cauchy.

For this, we recall a crucial lemma from [8], i.e. (Lemma 1.5), that specify when a b-rectangular Cauchy sequence can't have two limits in a b-g.m.s.

Lemma 1.5. Let $(X, d)$ be a b-rectangular metric space, with the coefficient $s \geq 1$. Let $\left(x_{n}\right)$ be a b-rectangular Cauchy sequence in $X$, such that $x_{n} \neq x_{m}$, for each $n \neq m$. Then $\left(x_{n}\right)$ can converge to at most one point.

Also, we recall from [12] and [8] the following crucial lemma.

Lemma 1.6. Let $(X, d)$ be a b-rectangular metric space, with the coefficient $s \geq 1$. Also, let $\left(x_{n}\right)$ be a sequence for which $x_{n} \neq x_{m}$, for every $n \neq m$, with $\lim _{n \rightarrow \infty} d\left(x_{n}, x_{n+1}\right)=0$. If $\left(x_{n}\right)$ is not a b-rectangular Cauchy sequence, then there exists $\varepsilon>0$, such that for each $k \in \mathbb{N}$, there exists $(m(k))$ and $(n(k))$ two sequences of positive integers, such that

$$
\begin{aligned}
& d\left(x_{m(k)}, x_{n(k)}\right) \geq \varepsilon, \\
& \frac{\varepsilon}{s} \leq \limsup _{k \rightarrow \infty} d\left(x_{m(k)}, x_{n(k)-2}\right) \leq \varepsilon \text { and } \\
& \frac{\varepsilon}{s} \leq \limsup _{k \rightarrow \infty} d\left(x_{m(k)+1}, x_{n(k)-1}\right) .
\end{aligned}
$$


In [22], another crucial lemma regarding sequences in b-rectangular metric spaces was presented. For convenience, we remind it below.

Lemma 1.7. Let $(X, d)$ be a b-g.m.s., with coefficient $s \geq 1$.

(a) Consider two sequences $\left(x_{n}\right)$ and $\left(y_{n}\right)$, such that $x_{n}$ converges to $x \in X$ and $y_{n}$ converges to $y \in X$, with $x \neq y$. Also, suppose that for each $n \in \mathbb{N}, x_{n} \neq x$ and $y_{n} \neq y$. Then

$$
\frac{1}{s} d(x, y) \leq \liminf _{n \rightarrow \infty} d\left(x_{n}, y_{n}\right) \leq \limsup _{n \rightarrow \infty} d\left(x_{n}, y_{n}\right) \leq s d(x, y) .
$$

(b) Consider an element $y \in X$ and a b-rectangular Cauchy sequence $\left(x_{n}\right)$, such that $x_{n} \neq x_{m}$, for each $n \neq m$. Moreover, suppose that the sequence $\left(x_{n}\right)$ converges to an element $x \neq y$. Then

$$
\frac{1}{s} d(x, y) \leq \liminf _{n \rightarrow \infty} d\left(x_{n}, y\right) \leq \limsup _{n \rightarrow \infty} d\left(x_{n}, y\right) \leq s d(x, y) .
$$

Finally, for the convenience of the reader, we recall some important results in brectangular metric spaces. In [9], George et.al.studied basic contraction-type mappings in b-rectangular metric spaces, like Kannan operators, i.e.

$$
d(T x, T y) \leq \lambda[d(x, T x)+d(y, T y)], \text { with } \lambda \in\left[0, \frac{1}{s+1}\right]
$$

In [8], Radenovic et.al. extended the results to mappings satisfying

$$
d(f x, g y) \leq a d(g x, g y)+b[d(g x, f x)+d(g y, f y)],
$$

for each $x, y \in X$ and studied unique coincidence and common fixed points for the pair of operators $(f, g)$ that satisfies some additional assumptions.

Also, for more results in b-rectangular metric spaces and for a consistent survey on different generalized metric-type spaces, we recommend [11] and [12].

Now, regarding generalized contraction mappings we recall some recent advances in this subfield of fixed point theory.

In [13], Karapinar studied unique fixed points for some generalized contractions on cone Banach spaces satisfying the following contractive-type conditions

$$
d(x, T x)+d(y, T y) \leq p d(x, y), \text { where } p \in[0,2)
$$

and

$$
a d(T x, T y)+b[d(x, T x)+d(y, T y)] \leq s d(x, y), \text { with } 0 \leq s+|a|-2 b<2(a+b) .
$$

Moreover, in 2009, Kumar [14] presented some theorems for two maps satisfying the following

$$
d(f x, f y) \geq q d(g x, g y), \text { with } q>1,
$$

where $\mathrm{f}$ is onto and $\mathrm{g}$ is one-to-one.

Moosaei, Azizi, Asadi and Wang generalized the results of Karapinar as follows In [15], Moosaei used Krasnoselskii's iteration defined in convex metric spaces, for the following mappings, that satisfy

$$
d(T x, T y)+d(x, T x)+d(y, T y) \leq r d(x, y), \text { where } r \in[2,5),
$$


respectively

$a d(x, f x)+b d(y, f y)+c d(f x, f y) \leq k d(x, y)$, with $2 b-|c| \leq k<2(a+b+c)-|c|$.

In [17], Moosaei and Azizi extended the results to generalized contraction-type operators, studying coincidence points for various mappings, such as

$$
a d(S x, T x)+b d(S y, T y)+c d(T x, T y) \leq e d(x, y)
$$

where $T(K) \subset S(K), K$ and $S(K)$ are closed and convex subsets of a convex metric space and the coefficients satisfy

$$
2 b-|c| \leq e<2(a+b+c)-|c| .
$$

Nevertheless, in 2014, Moosaei [16] studied a more generalized pair of contractions $(S, T)$, where

$$
\alpha d(T x, T y)+\beta[d(S x, T x)+d(S y, T y)]+\gamma[d(S x, T y)+d(S y, T x)] \leq \eta d(S x, S y)
$$

with some assumptions on contractive-coefficients, i.e.

$$
2 \beta+\gamma-|\gamma|-\alpha \leq \eta<\alpha+2 \beta+3 \gamma-|\gamma| \text { and } \beta+\gamma \leq 0
$$

Asadi in [3], using the same iteration (Krasnoselskii) on convex metric spaces, studied fixed points for generalized Hardy-Rogers type-mappings, as follows

$$
a d(x, T x)+b d(y, T y)+c d(T x, T y)+e d(T y, x)+f d(y, T x) \leq k d(x, y),
$$

where

$$
\frac{b+e-|f|(1-\lambda)-|c| \lambda}{1-\lambda} \leq k<\frac{a+b+c+e+f-|c| \lambda-|f|(1-\lambda)}{1-\lambda},
$$

and $\lambda \in[0,1]$ is the coefficient of Krasnoselskii's iteration.

Furthermore, Wang and Zhang, in [23] extended the above results for pairs of generalized Hardy-Rogers type contractions.

Now, expansive and expansive-type mappings can be considered a particular case of generalized contractions. Regarding the former ones, we recall some recent development into the study of this type of operators.

In 2011, Aage [1] considered expansive mappings in cone metric spaces. The more general form of these mappings, with some underlying assumptions, are

$$
d(T x, T y) \geq k d(x, y)+l d(x, T x)+p d(y, T y)
$$

where $T$ satisfies $K \geq-1, p<1, l>1$ and $k+l+p>1$.

Aydi et.al. studied in [4] some interesting fixed point theorems for pairs of expansive mappings for spaces endowed with c-distances. We recall them using the standard notations for metric spaces, i.e.

$$
d(T x, T y) \geq a d(f x, f y)+b d(T x, f x)+c d(T y, f y)
$$

with $b<1, a \neq 0, f(X) \subseteq T(X)$ and $(T(X), d) \subset(X, d)$ complete.

Also, in cone rectangular metric spaces, some fixed point theorems were developed. For example, in [20], pair of mappings satisfying

$$
d(f x, f y) \geq \alpha d(g x, g y)+\beta d(f x, g x)+\gamma d(f y, g y)
$$


were studied, with some assumptions on the coefficients $\alpha, \beta$ and $\gamma$ and on the range of $g$ and $f$.

These pairs of generalized mappings were extended by Olaoluwa and Olaleru in [18], but in the framework of b-metric spaces and for a pair of four mappings, as follows

$$
d(f x, g y) \geq a_{1} d(S x, T y)+a_{2} d(f x, S x)+a_{3} d(g y, T y)+a_{4} d(f x, T y)+a_{5} d(g y, S x) .
$$

Also, for the sake of convenience, we recall other studies in metric-type spaces and for expansive-type mappings, as follows: in [24] generalized mappings were studied on cone rectangular metric spaces using the technique of scalarizing, in [21] mappings that satisfy

$$
d(T x, T y) \leq \varphi(d(x, y))
$$

were studied on cone rectangular metric spaces and in [19], fixed point theorems for a general type of expansive mappings were developed, satisfying

$$
\phi\left(d\left(S^{2} x, T S y\right)\right) \geq \frac{1}{3}\left[d\left(S x, S^{2} x\right)+d(T S y, S y)+d(S x, S y)\right] .
$$

Also, in the context of dislocated metric spaces, Daheriya et.al. [7] studied rationaltype expansive mappings, and in [2] Alghamdi studied fixed points for generalized expansive mappings in b-metric like spaces.

The purpose of this work is to extend some fixed results for a hybrid class of generalized contractive-type mappings and for some expansive-type operators in the context of b-rectangular metric spaces. Moreover, at the end of the second section, we shall let and open problem.

\section{Main results}

Moosaei in [15] used Krasnoselskii iteration to develop fixed point theorems for generalized contractions on convex metric spaces. It is easily seen that we can use Picard instead of Krasnoselkii sequences in metric spaces.

In this section, our aim is to extend the results of Moosaei [15] for generalized contraction mappings from metric spaces to b-rectangular metric spaces. Also, we extend and develop the fixed point results of Aage [1] from cone metric spaces to b-g.m.s. Furthermore, we extend results from [20] of Patil, from rectangular metric spaces to b-rectangular ones (b-g.m.s).

Also, examples similar to those in [1], [12] and [20] justifying our theorems are given. Now, let's consider generalized contractions $f: X \rightarrow X$ on a b-g.m.s. $X$, satisfying the following condition:

$$
a d(x, f x)+b d(y, f y)+c d(f x, f y) \leq k d(x, y) .
$$

We will analyze two separate cases: when $c>0$ and $c<0$. Also, for expansive-type mappings, i.e. when $c<0$, we consider two types of sequence, namely the classical Picard iteration $x_{n+1}=f x_{n}$, for each $n \in \mathbb{N}$ and the 'inverse' Picard iteration, i.e. $x_{n}=f x_{n+1}$, for each $n \in \mathbb{N}$, for which we require that the operator $f$ is onto.

Our first result is a theorem for the existence and uniqueness of the fixed point of a mapping satisfying the contractive condition from above. The technique we will use is based on the (Lemma 1.6). 
Theorem 2.1. Let $(X, d)$ be a complete b-rectangular metric space (b-gms), with coefficient $s>1$. Consider a mapping $f: X \rightarrow X$, satisfying the following contractive condition

$$
a d(x, f x)+b d(y, f y)+c d(f x, f y) \leq k d(x, y), \text { where } 0 \leq k-b<\frac{a+c}{s} .
$$

Also, suppose the following assumptions are satisfied

(A) If $c>0$ and $k \geq 0$, then $\frac{k}{c}<\frac{1}{s}$,

(B) If $c>0$ and $k \leq 0$, then we have no additional conditions,

(C) If $c<0$ and $k<0$, then $\frac{k}{c}>s^{2}$.

Then, the Picard sequence $\left(x_{n}\right)$, defined as $x_{n+1}=f x_{n}$, for each $n \in \mathbb{N}$ converges to a fixed point of the mapping $f$.

Proof. We consider the Picard iterative process $\left(x_{n}\right)$, defined as $x_{n+1}=f x_{n}$, for each $n \in \mathbb{N}$. Applying the contractive condition for the pair $\left(x_{n-1}, x_{n}\right)$, we get that

$$
\begin{aligned}
& a d\left(x_{n}, f x_{n}\right)+b d\left(x_{n-1}, f x_{n-1}\right)+c d\left(f x_{n-1}, f x_{n}\right) \leq k d\left(x_{n-1}, x_{n}\right) \\
& a d\left(x_{n}, x_{n+1}\right)+b d\left(x_{n-1}, x_{n}\right)+c d\left(x_{n}, x_{n+1}\right) \leq k d\left(x_{n-1}, x_{n}\right) \\
& (a+c) d\left(x_{n}, x_{n+1}\right) \leq(k-b) d\left(x_{n-1}, x_{n}\right)
\end{aligned}
$$

So $d\left(x_{n}, x_{n+1}\right) \leq \delta d\left(x_{n-1}, x_{n}\right)$, where $\delta:=\frac{k-b}{a+c} \in\left[0, \frac{1}{s}\right)$ from the theorem's assumptions, since $0 \leq k-b<\frac{a+c}{s}$.

So $d\left(x_{n}, x_{n+1}\right) \leq \delta^{n} d\left(x_{0}, x_{1}\right)$. Since $\delta \in\left[0, \frac{1}{s}\right)$, it follows that $\lim _{n \rightarrow \infty} d\left(x_{n}, x_{n+1}\right)=0$. Also, by a routine argument (by reductio ad absurdum), it follows easily that $x_{n} \neq x_{n+1}$, for each $n \in \mathbb{N}$ and that $x_{n} \neq x_{m}$, for each $n \neq m$.

The next step is to show that the sequence $\left(x_{n}\right)$ is b-rectangular Cauchy. We will use (Lemma 1.6) and we shall apply it on three different cases

(1) Case $c>0$ : Let's suppose that the sequence $\left(x_{n}\right)$ is not b-rectangular Cauchy. Then, there exists $\varepsilon>0$ and two sequences of nonnegative real numbers $(m(k))$ and $(n(k))$, such that the assumptions from (Lemma 1.6) are satisfied.

Now, we will apply the contraction condition for $x=x_{m(k)}$ and $y=x_{n(k)-2}$. It follows that

$a d\left(x_{m(k)}, x_{m(k)+1}\right)+b d\left(x_{n(k)-2}, x_{n(k)-1}\right)+c d\left(x_{m(k)+1}, x_{n(k)-1}\right) \leq k d\left(x_{m(k)}, x_{n(k)-2}\right)$

$c d\left(x_{m(k)+1}, x_{n(k)-1}\right) \leq k d\left(x_{m(k)}, x_{n(k)-2}\right)-a d\left(x_{m(k)}, x_{m(k)+1}\right)-b d\left(x_{n(k)-2}, x_{n(k)-1}\right)$.

Because $c>0$, we have that

$d\left(x_{m(k)+1}, x_{n(k)-1}\right) \leq \frac{k}{c} d\left(x_{m(k)}, x_{n(k)-2}\right)-\frac{a}{c} d\left(x_{m(k)}, x_{m(k)+1}\right)-\frac{b}{c} d\left(x_{n(k)-2}, x_{n(k)-1}\right)$.

Now, we want to apply the limit superior. We make the following necessary remark and consider the following cases

If $a \geq 0$, then $-\frac{a}{c} \leq 0$, so $-\frac{a}{c} d\left(x_{m(k)}, x_{m(k)+1}\right) \leq 0$, so an upper bound for this element is 0 . 
If $a \leq 0$, then $-\frac{a}{c} \geq 0$, so $-\frac{a}{c} d\left(x_{m(k)}, x_{m(k)+1}\right) \geq 0$. Applying the limit superior, we get that

$$
\begin{aligned}
\limsup _{k \rightarrow \infty}\left(-\frac{a}{c}\right) d\left(x_{m(k)}, x_{m(k)+1}\right) & =\left(-\frac{a}{c}\right) \limsup _{k \rightarrow \infty} d\left(x_{m(k)}, x_{m(k)+1}\right) \\
& =\left(-\frac{a}{c}\right) \lim _{k \rightarrow \infty} d\left(x_{m(k)}, x_{m(k)+1}\right)=0 .
\end{aligned}
$$

The same reasoning can be made about the sign of the coefficient $b$ and about the limit superior of the sequence $\left(d\left(x_{n(k)-2}, x_{n(k)-1}\right)\right)$ as a subsequence of $\left(d\left(x_{n}, x_{n-1}\right)\right)$.

Case (A): When $k \geq 0$.

Since $k \geq 0$, we have that $\frac{k}{c} \geq 0$. We know that $\limsup _{k \rightarrow \infty} d\left(x_{m(k)}, x_{n(k)-2}\right) \leq \varepsilon$. Multiplying by $\left(\frac{k}{c}\right)$ and taking the limit superior, we get that

$$
\begin{aligned}
\limsup _{k \rightarrow \infty}\left(\frac{k}{c}\right) d\left(x_{m(k)}, x_{n(k)-2}\right) & =\limsup _{k \rightarrow \infty}\left|\frac{k}{c}\right| d\left(x_{m(k)}, x_{n(k)-2}\right) \\
& =\frac{k}{c} \limsup _{k \rightarrow \infty} d\left(x_{m(k)}, x_{n(k)-2}\right) \leq \frac{k}{c} \varepsilon .
\end{aligned}
$$

From (Lemma 1.6), it follows that $\frac{\varepsilon}{s} \leq \limsup _{k \rightarrow \infty} d\left(x_{m(k)+1}, x_{m(k)-1}\right) \leq \frac{k}{c} \varepsilon$, so $\frac{1}{s} \leq \frac{k}{c}$.

This is a contradiction with the assumption that in this case we have $\frac{k}{c}<\frac{1}{s}$.

Case (B): When $k \leq 0$.

In this case we have that $\frac{k}{c} \leq 0$, so $\frac{k}{c} d\left(x_{m(k)}, x_{n(k)-2}\right) \leq 0$, then we can take 0 as an upper bound for it. By (Lemma 1.6), we have that $\frac{\varepsilon}{s} \leq 0$. Since $\varepsilon>0$ and $s \geq 1$, we got a contradiction.

Now, in the two cases from above, we have shown that $\left(x_{n}\right)$ is b-rectangular Cauchy. Moreover, we have said that $x_{n} \neq x_{m}$, for each $n \neq m$.

Since $(X, d)$ is complete, it implies that there exists $u \in X$, such that $x_{n} \rightarrow u$, i.e.

$$
\lim _{n \rightarrow \infty} d\left(x_{n}, u\right)=0 .
$$

Now, we shall show that $u$ is a fixed point for $f$

$$
\begin{aligned}
d(u, f u) & \leq s\left[d\left(u, x_{n}\right)+d\left(x_{n}, x_{n+1}\right)+d\left(x_{n+1}, f u\right)\right] \\
& =s\left[d\left(u, x_{n}\right)+d\left(x_{n}, x_{n+1}\right)+d\left(f x_{n}, f u\right)\right]
\end{aligned}
$$

Since $c>0$, then

$$
d\left(f x_{n}, f u\right) \leq \frac{k}{c} d\left(x_{n}, u\right)-\frac{b}{c} d\left(x_{n}, x_{n+1}\right)-\frac{a}{c} d(u, f u) .
$$

So

$$
d(u, f u) \leq s\left[d\left(u, x_{n}\right)+d\left(x_{n}, x_{n+1}\right)+\frac{k}{c} d\left(x_{n}, u\right)-\frac{b}{c} d\left(x_{n}, x_{n+1}\right)-\frac{a}{c} d(u, f u)\right]
$$


Taking the limit when $n \rightarrow \infty$, we get

$$
\left(1+s \frac{a}{c}\right) d(u, f u) \leq 0
$$

so $(c+s a) d(u, f u) \leq 0$. Furthermore, since $c>0$ and $0<(a+c)<(a+c s)$, then $u$ is a fixed point for $f$.

(2) Case $c<0$ : We have that

$$
\begin{aligned}
& a d(x, f x)+b d(y, f y)+c d(f x, f y) \leq k d(x, y) \\
& c d(f x, f y) \leq k d(x, y)-a d(x, f x)-b d(y, f y)
\end{aligned}
$$

So

$$
d(f x, f y) \geq \frac{k}{c} d(x, y)-\frac{a}{c} d(x, f x)-\frac{b}{c} d(y, f y) .
$$

This is a case of expansive-type mapping. By (Lemma 1.6), there exists $\varepsilon>0$, such that for every $k \in \mathbb{N}$, there exists $(m(k)),(n(k))$ two sequences of nonnegative real numbers such that the assumptions in the already mentioned lemma are true. By b-rectangular inequality, we have that

$$
\begin{aligned}
& d\left(x_{n(k)-2}, x_{m(k)}\right) \leq s\left[d\left(x_{m(k)-1}, x_{n(k)-3}\right)+d\left(x_{n(k)-3}, x_{n(k)-2}\right)+d\left(x_{m(k)-1}, x_{m(k)}\right)\right] \\
& s d\left(x_{m(k)-1}, x_{n(k)-3}\right) \geq d\left(x_{n(k)-2}, x_{m(k)}\right)-s d\left(x_{n(k)-3}, x_{n(k)-2}\right)-s d\left(x_{m(k)-1}, x_{m(k)}\right)
\end{aligned}
$$

Dividing by $s \geq 1$, we obtain the following

$$
d\left(x_{m(k)-1}, x_{n(k)-3}\right) \geq \frac{1}{s} d\left(x_{n(k)-2}, x_{m(k)}\right)-d\left(x_{n(k)-3}, x_{n(k)-2}\right)-d\left(x_{m(k)-1}, x_{m(k)}\right) .
$$

Case $(\mathbf{C})$ : When $k<0$ : Here we have that $\frac{k}{c} \geq 0$. Multiplying by $\left(\frac{k}{c}\right)$, it implies that

$$
\begin{aligned}
\frac{k}{c} d\left(x_{m(k)-1}, x_{n(k)-3}\right) & \geq \frac{k}{c s} d\left(x_{n(k)-2}, x_{m(k)}\right)-\frac{k}{c} d\left(x_{n(k)-3}, x_{n(k)-2}\right) \\
& -\frac{k}{c} d\left(x_{m(k)-1}, x_{m(k)}\right) .
\end{aligned}
$$

Now, we apply the contractive condition for $x=x_{m(k)-1}$ and $y=x_{n(k)-3}$, i.e.

$d\left(x_{m(k)}, x_{n(k)-2}\right) \geq \frac{k}{c} d\left(x_{m(k)-1}, x_{n(k)-3}\right)-\frac{a}{c} d\left(x_{m(k)-1}, x_{m(k)}\right)-\frac{b}{c} d\left(x_{n(k)-3}, x_{n(k)-2}\right)$.

So, combining the above inequalities, we get that

$$
\begin{aligned}
d\left(x_{m(k)}, x_{n(k)-2}\right) & \geq \frac{k}{c s} d\left(x_{n(k)-2}, x_{m(k)}\right)-\frac{k}{c} d\left(x_{n(k)-3}, x_{n(k)-2}\right)-\frac{k}{c} d\left(x_{m(k)-1}, x_{m(k)}\right) \\
& -\frac{a}{c} d\left(x_{m(k)-1}, x_{m(k)}\right)-\frac{b}{c} d\left(x_{n(k)-3}, x_{n(k)-2}\right) .
\end{aligned}
$$


From the limit superior, we have get the following

$$
\begin{aligned}
\limsup _{k \rightarrow \infty}\left(-\frac{k}{c}\right) d\left(x_{n(k)-3}, x_{n(k)-2}\right) & =\frac{k}{c} \limsup _{k \rightarrow \infty}-d\left(x_{n(k)-3}, x_{n(k)-2}\right) \\
& =-\frac{k}{c} \liminf _{k \rightarrow \infty} d\left(x_{n(k)-3}, x_{n(k)-2}\right) \\
& =\left(-\frac{k}{c}\right) \lim _{k \rightarrow \infty} d\left(x_{n(k)-3}, x_{n(k)-2}\right)=0
\end{aligned}
$$

We have the same reasoning for $d\left(x_{m(k)-1}, x_{m(k)}\right)$, with coefficient $-\frac{k}{c}$. Also, for coefficients $a$ and $b$, we have that

If $a \geq 0$, then $-\frac{a}{c} \geq 0$, so $\left(-\frac{a}{c}\right) d\left(x_{m(k)-1}, x_{m(k)}\right) \geq 0$, so we can make the lower bound 0 .

If $a \leq 0$, then $-\frac{a}{c} \leq 0$, so $\left(-\frac{a}{c}\right) d\left(x_{m(k)-1}, x_{m(k)}\right) \leq 0$, so taking the limit superior, it follows that:

$$
\begin{aligned}
\limsup _{k \rightarrow \infty}\left(-\frac{a}{c}\right) d\left(x_{m(k)-1}, x_{m(k)}\right) & =\frac{a}{c} \limsup _{k \rightarrow \infty}-d\left(x_{m(k)-1}, x_{m(k)}\right) \\
& =-\frac{a}{c} \liminf _{k \rightarrow \infty} d\left(x_{m(k)-1}, x_{m(k)}\right) \\
& =-\frac{a}{c} \lim _{k \rightarrow \infty} d\left(x_{m(k)-1}, x_{m(k)}\right)=0
\end{aligned}
$$

Same remarks can be made about the coefficient $b$ and for $d\left(x_{n(k)-3}, x_{n(k)-2}\right)$.

By (Lemma 1.6), we get that

$$
\varepsilon \geq \limsup _{k \rightarrow \infty} d\left(x_{m(k)}, x_{n(k)-2}\right) \geq \frac{k}{c s} \limsup _{k \rightarrow \infty} d\left(x_{m(k)}, x_{n(k)-2}\right) \geq \frac{\varepsilon k}{c s^{2}} .
$$

So $\frac{1}{s^{2}} \leq \frac{c}{k}$. This is a contradiction with the fact that in this case $\frac{k}{c}>s^{2}$.

Now, since $x_{n} \neq x_{m}$, for each $n \neq m, d\left(x_{n}, x_{n+1}\right) \rightarrow 0,\left(x_{n}\right)$ Cauchy b-rectangular and $(X, d)$ is complete, then there exists $u \in X$, such that $x_{n} \rightarrow u$. We shall show that $u$ is a fixed point for the mapping $f$.

Applying the contractive condition on the pair $\left(u, x_{n}\right)$, we get

$$
\begin{aligned}
a d(u, f u)+b d\left(x_{n}, f x_{n}\right)+c d\left(f u, f x_{n}\right) & \leq k d\left(u, x_{n}\right) \\
a d(u, f u)+b d\left(x_{n}, x_{n+1}\right)+c d\left(f u, x_{n+1}\right) & \leq k d\left(u, x_{n}\right)
\end{aligned}
$$

Letting $n \rightarrow \infty$, we have $(a+c) d(u, f u) \leq 0$ and since we know that $a+c>0$, it follows that $u$ is a fixed point for the mapping $f$.

Relative to (Theorem 2.1), we give two examples that validate cases $(\mathrm{A})$ and $(\mathrm{C})$ : From [12], we recall an example of a complete b-rectangular metric space.

Example 2.2. Let $X=A \cup B$, where $A=\left\{\frac{1}{n} \mid n=\overline{2,5}\right\}$ and $B=[1,2]$. We define $d: X \times X \rightarrow[0, \infty)$, such that $d(x, y)=d(y, x)$ and

$$
d\left(\frac{1}{2}, \frac{1}{3}\right)=d\left(\frac{1}{4}, \frac{1}{5}\right)=\frac{3}{100},
$$




$$
\begin{aligned}
& d\left(\frac{1}{2}, \frac{1}{5}\right)=d\left(\frac{1}{3}, \frac{1}{4}\right)=\frac{2}{100}, \\
& d\left(\frac{1}{4}, \frac{1}{3}\right)=d\left(\frac{1}{5}, \frac{1}{3}\right)=\frac{6}{100},
\end{aligned}
$$

$d(x, y)=(x-y)^{2}$, otherwise.

Then $(X, d)$ is a complete b-rectangular metric space, with coefficient $s=3$. Furthermore, $(X, d)$ is not a metric space or a rectangular metric space.

Regarding case (A) of (Theorem 2.1), we give the following example.

Example 2.3. Let $(X, d)$ be the b-rectangular metric space defined above, with $s=3$. Also, define $f: X \rightarrow X$, such as

$$
f(x)=\left\{\begin{array}{l}
\frac{1}{3}, x \in A \\
\frac{1}{5}, x \in B
\end{array}\right.
$$

It is easy to observe that $f$ has a unique fixed point $\frac{1}{3}$. Moreover, we shall show that $f$ satisfies

$$
1 \cdot d(f x, f y) \leq \frac{1}{52} d(x, y)+\frac{1}{4} d(x, f x)+\frac{23}{100} d(y, f y)
$$

for each $x, y \in X$.

Let's define: $a=\frac{-1}{4}, b=\frac{-23}{100}, k=\frac{1}{52}, c=1$ and $s=3$.

We have the following cases
1) $x \in A$ and $y \in A: d(f x, f y)=d\left(\frac{1}{3}, \frac{1}{3}\right)=0$, so the above inequality is valid.
2) $x \in B$ and $y \in B: d(f x, f y)=d\left(\frac{1}{5}, \frac{1}{5}\right)=0$, so the inequality of $f$ is true.

Now, for the non-trivial cases, it follows that:

3) $x \in A$ and $y \in B$ :

$$
\begin{gathered}
d(f x, f y)=\left(\frac{1}{3}, \frac{1}{5}\right)=\frac{6}{100}, \\
d(x, f x)=d\left(x, \frac{1}{3}\right) \geq \min _{x \in A} d\left(x, \frac{1}{3}\right)=\frac{1}{200}, \\
d(y, f y)=d\left(y, \frac{1}{5}\right)=\left(y-\frac{1}{5}\right)^{2}=y^{2}-\frac{2}{5} y+\frac{1}{25} \geq \min _{y \in[1,2]}=1-\frac{1}{4}+\frac{1}{25}=\frac{6}{25} .
\end{gathered}
$$

Also $d(x, y)=(y-x)^{2}=|y-x|^{2}$.

We have that

$$
d(f x, f y) \leq k d(x, y)+(-a) \min _{x \in A} d(x, f x)+(-b) \min _{y \in B} d(y, f y) .
$$

So

$$
\frac{6}{100} \leq \frac{1}{52}|y-x|^{2}+\frac{1}{4} \cdot \frac{1}{200}+\frac{23}{100} \cdot \frac{6}{25}
$$


so $\frac{1}{52}|y-x|^{2} \geq \frac{-6619}{12000}$, which is obviously true.

4) $x \in B$ and $y \in A$

and

$$
\begin{gathered}
d(f x, f y)=\left(\frac{1}{3}, \frac{1}{5}\right)=\frac{6}{100}, \\
d(x, f x) \geq \min _{x \in B} d(x, f x)=\frac{6}{25}, \\
d(y, f y) \geq \min _{y \in A}=\frac{1}{200}
\end{gathered}
$$

$$
d(x, y)=(y-x)^{2}=|y-x|^{2} .
$$

We have that

so $\frac{1}{52}|y-x|^{2} \geq \frac{-419}{6000}$, which is also true.

$$
\frac{6}{100} \leq \frac{1}{52}|y-x|^{2}+\frac{1}{4} \cdot \frac{6}{25}+\frac{23}{100} \cdot \frac{1}{200},
$$

Moreover, we show that the conditions from (Theorem 2.1) - case (A) on the coefficients are satisfied

$$
\begin{aligned}
& c>0 \Leftrightarrow 1>0 \\
& k>0 \Leftrightarrow \frac{1}{52}>0 \\
& a+c=1-\frac{1}{4}=\frac{3}{4}>0 \\
& b \leq k \Leftrightarrow-\frac{23}{100} \leq \frac{1}{52} \\
& \frac{k}{c}<\frac{1}{s} \Leftrightarrow k<\frac{1}{3} \Leftrightarrow 3<52 \\
& k<b+\frac{a+c}{s} \Leftrightarrow \frac{1}{52}+\frac{23}{100}<\frac{1}{4} \Leftrightarrow 324<325
\end{aligned}
$$

Now, we construct an example of a complete b-rectangular metric space, which will be used further in this section.

Example 2.4. Let $X=\{1,2,3,4\}$ and define $d: X \times X \rightarrow[0, \infty)$, such as

$$
\begin{aligned}
& d(1,2)=d(2,1)=\frac{6}{10} \\
& d(1,3)=d(3,1)=\frac{1}{10} \\
& d(2,3)=d(3,2)=\frac{1}{10} \\
& d(1,4)=d(4,1)=d(2,4)=d(4,2)=d(3,4)=d(4,3)=\frac{2}{10}
\end{aligned}
$$

We will prove that $(X, d)$ is a b-rectangular metric space with coefficient $s=\frac{3}{2}$, which is not a rectangular metric space. 
For a b-rectangular metric space, we have that $d(x, y) \leq s[d(x, u)+d(u, v)+d(v, y)]$, for each $u, v \notin\{x, y\}$, with $u, v$ being distinct. We have the following cases.

- When $x=y$, the right hand side is 0 , so the above inequality remains valid.

- When $x \neq y$, we employ the following sub-cases

Case (1): If $x=1$ and $y=2(x=2$ and $y=1$ by symmetry):

$$
\begin{aligned}
\frac{6}{10} & \leq s[d(1, u)+d(u, v)+d(v, 2)], \text { for } u, v \notin\{1,2\}, \text { i.e. } u, v \in I_{1}=\{3,4\} \\
\frac{6}{10} & =d(1,2) \leq s\left[\min _{u \in I_{1}} d(1, u)+d(3,4)+\min _{v \in I_{1}} d(v, 2)\right] \\
\frac{6}{10} & \leq s\left[\frac{1}{10}+\frac{2}{10}+\frac{1}{10}\right], \text { so } s \geq \frac{3}{2}
\end{aligned}
$$

Case (2): If $x=3$ and $y=1(x=1$ and $y=3$ by symmetry):

$$
\begin{aligned}
\frac{1}{10} & \leq s[d(3, u)+d(u, v)+d(v, 1)], \text { for } u, v \notin\{1,3\}, \text { i.e. } u, v \in I_{2}=\{2,4\} \\
\frac{1}{10} & =d(3,1) \leq s\left[\min _{u \in I_{2}} d(3, u)+d(2,4)+\min _{v \in I_{2}} d(v, 1)\right] \\
\frac{1}{10} & \leq s\left[\frac{1}{10}+\frac{2}{10}+\frac{1}{10}\right], \text { so } s \geq \frac{1}{4}
\end{aligned}
$$

Case (3): If $x=4$ and $y=1$ ( $x=1$ and $y=4$ by symmetry):

$$
\begin{aligned}
\frac{2}{10} & \leq s[d(3, u)+d(u, v)+d(v, 1)], \text { for } u, v \notin\{1,4\}, \text { i.e. } u, v \in I_{3}=\{2,3\} \\
\frac{2}{10} & =d(4,1) \leq s\left[\min _{u \in I_{3}} d(4, u)+d(2,3)+\min _{v \in I_{3}} d(v, 1)\right] \\
\frac{2}{10} & \leq s\left[\frac{2}{10}+\frac{1}{10}+\frac{1}{10}\right], \text { so } s \geq \frac{1}{2}
\end{aligned}
$$

Case (4): If $x=2$ and $y=4(x=4$ and $y=2$ by symmetry):

$$
\begin{aligned}
& \frac{2}{10} \leq s[d(2, u)+d(u, v)+d(v, 4)], \text { for } u, v \notin\{2,4\}, \text { i.e. } u, v \in I_{4}=\{1,3\} \\
& \frac{2}{10}=d(4,2) \leq s\left[\min _{u \in I_{4}} d(2, u)+d(1,3)+\min _{v \in I_{4}} d(v, 4)\right] \\
& \frac{2}{10} \leq s\left[\frac{1}{10}+\frac{1}{10}+\frac{2}{10}\right], \text { so } s \geq \frac{1}{2}
\end{aligned}
$$

Case (5): If $x=3$ and $y=4(x=4$ and $y=3$ by symmetry):

$$
\begin{aligned}
& \frac{2}{10} \leq s[d(3, u)+d(u, v)+d(v, 4)], \text { for } u, v \notin\{3,4\}, \text { i.e. } u, v \in I_{5}=\{1,2\} \\
& \frac{2}{10}=d(3,4) \leq s\left[\min _{u \in I_{5}} d(3, u)+d(1,2)+\min _{v \in I_{5}} d(4, v)\right] \\
& \frac{2}{10} \leq s\left[\frac{1}{10}+\frac{6}{10}+\frac{2}{10}\right], \text { so } s \geq \frac{2}{9}
\end{aligned}
$$


So $s \geq \frac{3}{2}>1$, so we can take $s=\frac{3}{2}$.

Furthermore, $(X, d)$ is not a b-g.m.s., because

$$
\frac{6}{10}=d(1,2)>d(1,3)+d(3, u)+d(u, 2)=\frac{1}{10}+\frac{2}{10}+\frac{2}{10}=\frac{5}{10},
$$

so $6>5$, which is valid.

Now, we construct an example, justifying case $(C)$ of (Theorem 2.1).

Example 2.5. Let $X=\{1,2,3,4\}$ the b-rectangular metric space defined above, with coefficient $s=\frac{3}{2}$.

Let $f(x)=\left\{\begin{array}{ll}3, & x \neq 4 \\ 1, & x=4\end{array}\right.$ a self-mapping defined on $X$.

We shall show that $f$ satisfies

$$
d(f x, f y) \geq(-3) d(x, y)-5 d(x, f x)+3 d(y, f y)
$$

and also the conditions from case $(C)$ of (Theorem 2.1).

Let $f$ satisfy $c d(f x, f y) \geq k d(x, y)-a d(x, f x)-b d(y, f y)$. Let's normalize the contractive condition, by taking $c=-1<0$ We shall determine the coefficients $k, a, b$, with $k<0, a>0$ and $b<0$. We have the following cases

1 ) If $x=y$, then $d(f x, f y)=d(f x, f x)=0$, so the left hand side is 0 . Now, the right hand side is $k \cdot 0-a d(x, f x)-b d(x, f x)=-(a+b) d(x, f)$. This implies that $(a+b) d(x, f x) \geq 0$. We have two sub-cases:

If $x=3$, then $d(x, f x)=d(3,3)=0$, so the inequality is valid. Also, if $\neq 3$, then $d(x, f x)>0$, so we have the condition that $-b \leq a$.

2) If $\neq y$, we have the following sub-cases

a) For $x=4$ and $y \neq 4$, it follows that $d(f y, f x)=d(f y, 1)$. Since $y \neq 4$, then $f y=3$, so $d(f x, f y)=d(1,3)=\frac{1}{10}$.

Moreover, one can easily verify that $d(x, y)=d(4, y)=\frac{2}{10}$, for each $y \neq 4$, $d(x, f x)=d(4, f x)=\frac{2}{10}$, for each $x \in X$ and $d(y, f y)=d(y, 3) \leq \max _{y \neq 4} d(y, 3)=\frac{2}{10}$.

b) For $y=4$ and $x \neq 4$, it follows that $d(f x, f y)=\frac{1}{10}$.

Moreover, we have that $d(x, y)=d(4, x)=\frac{2}{10}$, for each $x \neq 4$,

$d(x, f x)=d(x, 3)=\geq \min _{x \neq 4} d(x, 3)=\frac{1}{10}$ and $d(y, f y)=d(4, f y)=\frac{2}{10}$, for each value of $f y$.

c) For $y \neq y \neq 4$ (simultaneously), it follows that $d(f x, f y)=d(3,3)=0$. Also

$$
k d(x, y)-a d(x, f x)-b d(y, f y) \leq 0, \quad \text { so } \quad k d(x, y)-b d(x, y) \leq a d(x, f x) .
$$


Now $d(x, y) \geq \min _{x, y \in X} d(x, y)=\frac{1}{10}$.

Furthermore, we have that

$$
d(y, f y)=d(y, 3) \leq \max _{y \neq 4} d(y, 3)=\frac{2}{10} \quad \text { and } \quad d(x, f x)=d(x, 3) \geq \min _{x \neq 4} d(x, 3)=\frac{1}{10} .
$$

Now, we analyze the conditions on $f$.

For the case (1), we get $-b \leq a$. For the case $(2 \mathrm{a})$, we get that

$$
\begin{aligned}
d(f x, f y) & =\frac{1}{10} \geq k d(x, y)-a d(x, f x)-b \max _{y \neq 4} d(y, f y) \\
& =\frac{2 k}{10}-\frac{2 a}{10}-\frac{2 b}{10} \geq \frac{2 k}{10}-\frac{2 a}{10}-b d(y, f y)
\end{aligned}
$$

because $b<0$. So $k<a+b+\frac{1}{2}$.

For the case $(2 \mathrm{~b})$, we obtain

$$
\begin{aligned}
d(f x, f y) & =\frac{1}{10} \geq k d(x, y)-a \min _{x \neq 4} d(x, f x)-b d(y, f y) \\
& =\frac{2 k}{10}-\frac{a}{10}-\frac{2 b}{10} \geq \frac{2 k}{10}-\frac{2 b}{10}-a d(x, f x)
\end{aligned}
$$

because $a>0$. So $k<\frac{a}{2}+b+\frac{1}{2}$.

For the case $(2 \mathrm{c})$, it follows that

$$
\begin{aligned}
d(f x, f y) & =0 \geq k \min _{x \neq y \neq 4} d(x, y)-a \min _{x \neq 4} d(x, f x)-b \max _{y \neq 4} d(y, f y) \\
& =\frac{k}{10}-\frac{a}{10}-\frac{2 b}{10} k d(x, y)-a d(x, f x)-b d(y, f y),
\end{aligned}
$$

because $b, k<0$ and $a>0$, so $k-s b \leq a$.

Additionally, $f$ satisfies the conditions from (Theorem 2.1) - Case (C).

Let's take $k=-3, c=-1, a=5, b=-3$, with $s=\frac{3}{2}$. We verify that the coefficients $a, b, c, k$ verify all of the above conditions

$$
\left\{\begin{array}{l}
-b \leq a \Leftrightarrow 3 \leq 5, k<a+b+\frac{1}{2} \Leftrightarrow-3<2+\frac{1}{2} \\
k<\frac{a}{2}+b+\frac{1}{2} \Leftrightarrow 10+\frac{1}{2}>0, k-2 b \leq a \Leftrightarrow 3>1 \\
b \leq k \Leftrightarrow-3 \leq-3, \frac{k}{c}>s^{2} \Leftrightarrow 12>9 \\
k<b+\frac{a+c}{s} \Leftrightarrow 6>0, a+c>0 \Leftrightarrow 6>0
\end{array}\right.
$$

Remark 2.6. We observe that the contractive condition when $c>0$, can be written as:

$$
d(f x, f y) \leq \frac{k}{c} d(x, y)-\frac{a}{c} d(x, f x)-\frac{b}{c} d(y, f y), \text { for each } x, y \in X .
$$

Taking $k>0, a<0$ and $b<0$, it follows that the operator $f$ is of Reich-type, so the above theorem (when $k>0$ ) is similar with the results of [8]. 
Now, we present an useful lemma for expansive-type mappings in b-rectangular metric spaces, following the technique used in [18].

Lemma 2.7. Let $(X, d)$ a b-rectangular metric space. Also, consider $\lambda \in \mathbb{R}$ and $x, y, z, w$ arbitrary elements of $X$, each distinct from each other. Then

$$
\begin{aligned}
\lambda d(x, z) & \geq\left[\frac{1+s^{2}}{2 s} \lambda+\frac{1-s^{2}}{2 s}|\lambda|\right] d(x, y)+\left[\frac{s-1}{2} \lambda-\frac{s+1}{2}|\lambda|\right] d(z, w) \\
& +\left[\frac{s-1}{2} \lambda-\frac{s+1}{2}|\lambda|\right] d(w, y) .
\end{aligned}
$$

Proof. Let $x, y, z, w$ arbitrary points from $X$, each distinct from each other. We analyze two cases for the parameter $\lambda \in \mathbb{R}$ :

Case (1): Let $\lambda \geq 0$. From the b-rectangular inequality, we get that:

$$
\begin{aligned}
& d(x, y) \leq s[d(x, z)+d(z, w)+d(w, y)] \\
& s d(x, z) \geq d(x, y)-s d(z, w)-s d(w, y) \\
& d(x, z) \geq \frac{1}{s} d(x, y)-d(z, w)-d(w, y) \\
& \lambda d(x, z) \geq \frac{\lambda}{s} d(x, y)-\lambda d(z, w)-\lambda d(w, y)
\end{aligned}
$$

Case (2): Let $\lambda \leq 0$. From the b-rectangular inequality, it follows that:

$$
\begin{aligned}
& d(x, z) \leq s[d(x, y)+d(y, w)+d(w, z)] \\
& \lambda d(x, z) \geq \lambda s d(x, y)+\lambda s d(y, w)+\lambda s d(w, z)
\end{aligned}
$$

So, from the above inequality, we have that

$$
\left\{\begin{array}{lll}
\lambda d(x, z) \geq \frac{\lambda}{s} d(x, y)-\lambda d(z, w)-\lambda d(w, y), & & \lambda \geq 0 \\
\lambda d(x, z) \geq \lambda s d(x, y)+\lambda s d(y, w)+\lambda s d(w, z), & & \lambda \leq 0
\end{array}\right.
$$

Combining these cases, it follows that

$\lambda d(x, z) \geq \varphi(\lambda) d(x, y)+\psi(\lambda) d(z, w)+\psi(\lambda) d(w, y)$, where

$$
\varphi(\lambda):=\left\{\begin{array}{ll}
\frac{\lambda}{s}, & \lambda \geq 0 \\
s \lambda, & \lambda \leq 0
\end{array} \text { and } \psi(\lambda):= \begin{cases}-\lambda, & \lambda \geq 0 \\
s \lambda, & \lambda \leq 0\end{cases}\right.
$$

Similar to [18], we get that

$$
\left\{\begin{array}{l}
\varphi(\lambda):=\frac{1+s^{2}}{2 s} \lambda+\frac{1-s^{2}}{2 s}|\lambda| \\
\psi(\lambda):=\frac{s-1}{2} \lambda-\frac{s+1}{2}|\lambda|
\end{array}\right.
$$

Also, as a final remark, we observe that $\psi(\lambda) \leq 0$, for each $\lambda \in \mathbb{R}$. remark.

For expansive-type mappings, i.e. when $c<0$, we make the following important 
Remark 2.8. We have studied contraction-type mappings, that satisfied

$$
\begin{aligned}
& a d(x, f x)+b d(y, f y)+c d(f x, f y) \leq k d(x, y) \\
& c d(f x, f y) \leq k d(x, y)-a d(x, f x)-b d(y, f y) \\
& d(f x, f y) \geq \frac{k}{c} d(x, y)-\frac{a}{c} d(x, f x)-\frac{b}{c} d(y, f y)
\end{aligned}
$$

By some substitutions we can make the mapping $f$ satisfy

$$
d(f x, f y) \geq \alpha d(x, y)+\beta d(x, f x)+\gamma d(y, f y)
$$

where

$$
\left\{\begin{array}{l}
\alpha=\frac{k}{c} \\
\beta=-\frac{a}{c} \\
\gamma=-\frac{b}{c}
\end{array}\right.
$$

We will analyze the cases when $\alpha \leq 0$ and $\alpha \geq 0$, so, when $k \geq 0, c<0$, respectively $k \leq 0, c<0$.

Now, involving rate of convergence, we present a constructive fixed point theorem for expansive-type mappings in b-rectangular metric spaces, using Picard iterative process.

Theorem 2.9. Let $(X, d)$ a complete b-rectangular metric space, endowed with coeffcient $s \geq 1$. Also, consider $f: X \rightarrow X$ a mapping satisfying

$$
d(f x, f y) \geq \alpha d(x, y)+\beta d(x, f x)+\gamma d(y, f y), \text { for each } x, y \in X .
$$

Moreover, suppose the following conditions are satisfied

(i) $\beta<1-s, \gamma>s, \alpha+\gamma<\frac{1-\beta}{s}$,

(ii) If $\alpha>\gamma$, then we have the additional assumptions $\alpha+1<\gamma\left(1+\frac{1}{s}\right)$.

If $\alpha<\gamma$, then we have the additional assumptions $\alpha>1$ and $1-\alpha<\gamma\left(\frac{1}{s}-1\right)$.

Then, the mapping $f$ has a fixed point.

Proof. In the proof of (Theorem 2.1), we have shown that the Picard sequence for generalized contraction satisfy $d\left(x_{n}, x_{n+1}\right) \leq \delta d\left(x_{n-1}, x_{n}\right)$, for each $n \in \mathbb{N}$, where $\delta=\frac{k-b}{a+c}$. This is also valid for the situation of expansive-type mappings, when $c<0$. The condition that the Picard sequence is asymptotically regular was that $0 \leq k-b<\frac{a+c}{s}$.

In our case,

$$
\delta=\frac{k-b}{a+c}=\frac{\frac{k}{c}-\frac{b}{c}}{\frac{a}{c}+1}=\frac{\alpha+\gamma}{1-\beta}
$$


Now $\delta \in\left[0, \frac{1}{s}\right)$, by hypothesis assumptions: $\beta<1, \alpha+\gamma>0$ and $\alpha+\gamma<\frac{1-\beta}{s}$.

By the contractive-type condition, we have that

$$
d(f x, f y) \geq \alpha d(x, y)+\beta d(x, f x)+\gamma d(y, f y)
$$

and applying it for the pair $\left(x_{n-1}, x_{n+1}\right)$, we obtain

$$
d\left(x_{n}, x_{n+2}\right) \geq \alpha d\left(x_{n-1}, x_{n+1}\right)+\beta d\left(x_{n-1}, x_{n}\right)+\gamma d\left(x_{n+1}, x_{n+2}\right)
$$

Now, we will try to evaluate an upper bound for $d\left(x_{n}, x_{n+2}\right)$, for each $n \in \mathbb{N}$, i.e. using (Lemma 2.7), we obtain that

$$
\gamma d\left(x_{n+1}, x_{n+2}\right) \geq \varphi(\gamma) d\left(x_{n}, x_{n+2}\right)+\psi(\gamma) d\left(x_{n}, x_{n-1}\right)+\psi(\gamma) d\left(x_{n-1}, x_{n+1}\right) .
$$

Now, let's denote by $d_{n}^{*}:=d\left(x_{n}, x_{n+2}\right)$ and by $d_{n}:=d\left(x_{n-1}, x_{n}\right)$, for each $n \in \mathbb{N}$.

From (2.1) we have

$$
d_{n}^{*} \geq \alpha d_{n-1}^{*}+\beta d_{n}+\varphi(\gamma) d_{n}^{*}+\psi(\gamma) d_{n}+\psi(\gamma) d_{n-1}^{*} .
$$

This means that

$$
[\varphi(\gamma)-1] d_{n}^{*} \leq[-\psi(\gamma)-\alpha] d_{n-1}^{*}+[-\psi(\gamma)-\beta] d_{n} \leq|\psi(\gamma)+\alpha| d_{n-1}^{*}+|\psi(\gamma)+\beta| d_{n}
$$

Let's denote by $a_{2}:=\frac{|\alpha+\psi(\gamma)|}{\varphi(\gamma)-1}$ and by $a_{1}:=\frac{|\beta+\psi(\gamma)|}{\varphi(\gamma)-1}$.

From the hypothesis, we know that $\varphi(\gamma)>1$, i.e. $\gamma>s>0$, since $\varphi(\gamma)=\frac{\gamma}{s}$. Then it follows that $a_{1}$ and $a_{2}$ are positive.

Furthermore, since $\gamma>0$, we have that $\psi(\gamma)=-\gamma<0$. So $a_{2}=\frac{|\alpha-\gamma|}{\frac{\gamma}{s}-1}$. For $a_{2}<1$, we get that $|\alpha-\gamma|<\frac{\gamma}{s}-1$. So, we have two cases:

- When $\alpha>\gamma$, i.e. $\alpha \stackrel{s}{-} \gamma>0$ :

Then, the condition that $a_{2}<1$ becomes $\alpha+1<\frac{\gamma}{s}+\gamma$, i.e. $\alpha+1<\gamma\left(1+\frac{1}{s}\right)$.

Now, since $\gamma+1<\alpha+1<\gamma\left(1+\frac{1}{s}\right)$, then $s<\gamma$, which is true. Also, since $\gamma+1<\alpha+1<\gamma\left(1+\frac{1}{s}\right)<2 \gamma$, then $1<\gamma$, which is a valid assumption.

Moreover, from the hypothesis condition that $\alpha+\gamma<\frac{1-\beta}{s}$, we employ two sub-cases If $\beta>0$, then $1-\beta<1$, i.e. $\alpha+\gamma<\frac{1}{s}<1$, so $\alpha+\gamma<1$. Since $\alpha, \gamma>s>1$, this is obviously not true.

If $\beta<0$, then $\beta<1$, so $1-\beta>0$ (the denominator in $\delta$ is positive, so $\delta$ is positive). Since $\beta<0$, then $\frac{1-\beta}{s}>\frac{1}{s}$. Moreover, since $\alpha+\gamma>1$, then we get $\beta<1-s$, which is valid from hypothesis (ii).

Finally, we can verify easily that since $s>1$, then $\beta<1$ and since $1-s<1$, then $s>0$, which are evidently true.

- We know verify the case when $\alpha<\gamma$, i.e. $\alpha-\gamma<0$ : 
Since $|\alpha-\gamma|=\gamma-\alpha<\frac{\gamma}{s}-1$, then $1-\alpha<\gamma\left(\frac{1}{s}-1\right)$, which is true by hypothesis (ii).

Moreover, since $\frac{1}{s}-1<0$, then $\alpha>1$ is obviously true, also by hypothesis. Also, since $\gamma>\alpha>1$, then $\gamma>1$, which is valid by the fact that $\gamma>s$.

Also, as in previous case, by the assumption on $\delta$ that $\alpha+\gamma<\frac{1-\beta}{s}$, if $\beta>0$, then $\alpha+\gamma<\frac{1-\beta}{s}<1$, which contradicts the fact that $\alpha, \gamma>1$.

So $\beta<0$ and from the assumption that $\beta<1-s$ means that the right hand side $\frac{1-\beta}{s}>1$, so $1<\alpha+\gamma<\frac{1-\beta}{s}$, which is valid.

So ${ }^{s} d_{n}^{*} \leq a_{2} d_{n-1}^{*}+a_{1} d_{n}$, for each $n \in \mathbb{N}$. We know that

$$
d_{n}=d\left(x_{n-1}, x_{n}\right) \leq \delta d\left(x_{n-1}, x_{n-2}\right) \leq \ldots \leq \delta^{n-1} D_{0},
$$

where $D_{0}:=d_{1}=d\left(x_{0}, x_{1}\right)$, with $x_{0}$ an arbitrary fixed element.

So $d_{n}^{*} \leq a_{2} d_{n-1}^{*}+a_{1} \delta^{n-1} D_{0}$.

We take a major bound for $d_{n}^{*}$ :

$$
\begin{aligned}
d_{n}^{*} & \leq a_{2} d_{n-1}^{*}+a_{1} \delta^{n-1} D_{0} \leq a_{2}\left(a_{2} d_{n-2}^{*}+a_{1} \delta^{n-2} D_{0}\right)+a_{1} \delta^{n-1} D_{0} \\
& =a_{2}^{2} d_{n-2}^{*}+a_{2} a_{1} \delta^{n-2} D_{0}+a_{1} \delta^{n-1} D_{0} \\
& \leq a_{2}^{2}\left(a_{2} d_{n-3}^{*}+a_{1} \delta^{n-3} D_{0}\right)+a_{1} a_{2} \delta_{n-2} D_{0}+a_{1} \delta^{n-1} D_{0} \\
& =a_{2}^{3} d_{n-3}^{*}+D_{0} a_{1}\left(\delta^{n-1}+a_{2} \delta^{n-2}+a_{2}^{2} \delta^{n-3}\right) D_{0} \leq \ldots \\
& \leq a_{2}^{k} d_{n-k}^{*}+a_{1}\left(\delta^{n-1}+a_{2} \delta^{n-2}+\ldots+a_{2}^{k-1} \delta^{n-k}\right) D_{0}
\end{aligned}
$$

The last term is $d_{0}^{*}=d\left(x_{2}, x_{0}\right)$, so $n-k=0 \Longrightarrow k=n$. This means that

$$
d_{n}^{*} \leq a_{2}^{n} d_{0}^{*}+a_{1} D_{0}\left(a_{2}^{0} \delta^{n-1}+a_{2} \delta^{n-2}+\ldots+a_{2}^{n-1} \delta^{0}\right)
$$

Let's denote by $S:=a_{2}^{0} \delta^{n-1}+a_{2} \delta^{n-2}+\ldots+a_{2}^{n-1} \delta^{0}$. The first term in the sum is $\delta^{n-1}$. This is a geometric progression, with general term $b_{n}$ and $\frac{b_{3}}{b_{2}}=a_{2} \frac{\delta^{n-3}}{\delta^{n-2}}=\frac{a_{2}}{\delta}$, SO

$$
S=\frac{\delta^{n-1} \cdot\left(1-\left(\frac{a_{2}}{\delta}\right)^{n}\right)}{1-\left(\frac{a_{2}}{\delta}\right)}=\frac{\delta^{n}-a_{2}^{n}}{\delta-a_{2}} .
$$

So $d_{n}^{*} \leq a_{2}^{n} d_{0}^{*}+\frac{\delta^{n}-a_{2}^{n}}{\delta-a_{2}} a_{1} D_{0}$. Now we can show that the sequence $\left(x_{n}\right)$ is b-rectangular Cauchy. We shall evaluate $d\left(x_{n}, x_{n+p}\right)$, for each $n \in \mathbb{N}$ and $p>0$ fixed. We divide in two cases: the first one, when $p=2 m$, with $m \geq 2$ and the second one, when $p=2 m+1$, with $m \geq 1$ :

Case (i): When $p=2 m+1$, with $m \geq 1$. We evaluate

$$
\begin{aligned}
& d\left(x_{n}, x_{n+p}\right)=d\left(x_{n}, x_{n+2 m+1}\right) \leq s\left[d\left(x_{n}, x_{n+1}\right)+d\left(x_{n+1}, x_{n+1}\right)+d\left(x_{n+2}, x_{n+2 m+1}\right)\right] \\
& \leq s\left[d_{n+2}+d_{n+1}\right]+s^{2}\left[d\left(x_{n+2}, x_{n+3}\right)+d\left(x_{n+3}, x_{n+4}\right)+d\left(x_{n+4}, x_{n+2 m+1}\right)\right] \\
& \leq s\left[d_{n+2}+d_{n+1}\right]+s^{2}\left[d_{n+3}+d_{n+4}\right]+s^{3}\left[d_{n+5}+d_{n+6}\right]+\ldots+s^{m} d_{n+2 m},
\end{aligned}
$$


where $d_{n+2 m}=d\left(x_{n+2 m}, x_{n+2 m+1}\right)$. So, we get the following estimation

$$
\begin{aligned}
d\left(x_{n}, x_{n+2 m+1}\right) & \leq s\left[\delta^{n} D_{0}+\delta^{n+1} D_{0}\right]+s^{2}\left[\delta^{n+2} D_{0}+\delta^{n+3} D_{0}\right] \\
& +s^{3}\left[\delta^{n+4} D_{0}+\delta^{n+5} D_{0}\right]+\ldots+s^{m} \delta^{n+2 m} D_{0} \\
& \leq s \delta^{n}\left[1+s \delta^{2}+s^{2} \delta^{4}+\ldots+\right] D_{0}+s \delta^{n+1}\left[1+s \delta^{2}+s^{2} \delta^{4}+\ldots+\right] D_{0} \\
& =\frac{1+\delta}{1-s \delta^{2}} s \delta^{n} D_{0},
\end{aligned}
$$

and by hypothesis we know that $s \delta^{2}<1$ is satisfied. So, $d\left(x_{n}, x_{n+2 m+1}\right) \rightarrow 0$, when $n \rightarrow \infty$ and $m \geq 1$ fixed.

Case (ii): When $p=2 m$, with $m \geq 2$. We evaluate

$$
\begin{aligned}
d\left(x_{n}, x_{n+2 m}\right) & \leq s\left[d\left(x_{n}, x_{n+1}\right)+d\left(x_{n+1}, x_{n+2}\right)+d\left(x_{n+2}, x_{n+2 m}\right)\right] \\
& \leq s\left[d_{n+2}+d_{n+1}\right]+s d\left(x_{n+2}, x_{n+2 m}\right) \\
& \leq s\left[d_{n+2}+d_{n+1}\right]+s^{2}\left[d_{n+4}+d_{n+3}\right]+s^{3}\left[d_{n+6}+d_{n+5}\right]+\ldots+ \\
& +s^{m-1}\left[d_{2 m-3}+d_{2 m-2}\right]+s^{m-1} d\left(x_{n+2 m-2}, x_{n+2 m}\right) \\
& \leq s\left[\delta^{n} D_{0}+\delta^{n+1} D_{0}\right]+s^{2}\left[\delta^{n+2} D_{0}+\delta^{n+3} D_{0}\right]+\ldots+ \\
& +s^{m-1}\left[\delta^{2 m-4} D_{0}+\delta^{2 m-3} D_{0}\right]+s^{m-1} d\left(x_{n+2 m-2}, x_{n+2 m}\right) \\
& \leq s \delta^{n}\left[1+s \delta^{2}+s^{2} \delta^{4}+\ldots\right] D_{0} \\
& +s \delta^{n+1}\left[1+s \delta^{2}+s^{2} \delta^{4}+\ldots\right] D_{0}+s^{m-1} d_{n+2 m}^{*} \\
& =\frac{1+\delta}{1-s \delta^{2}} s \delta^{n} D_{0}+s^{m-1} d_{n+2 m}^{*}
\end{aligned}
$$

Also, we have shown that $d_{n}^{*} \leq a_{2}^{n} d_{0}^{*}+\frac{\delta^{n}-a_{2}^{n}}{\delta-a_{2}} a_{1} D_{0}$. So $d_{n+2 m}^{*} \leq a_{2}^{n+2 m} d_{0}^{*}+Q a_{1} D_{0}$, where $Q:=\frac{\delta^{n+2 m}-a_{2}^{n+2 m}}{\delta-a_{2}}$.

Now, we have two cases: if $\delta-a_{2}>0$, then $Q=\frac{\delta^{n+2 m}-a_{2}^{n+2 m}}{\delta-a_{2}} \leq \frac{\delta^{n+2 m}}{\delta-a_{2}}$ and this converge to 0 as $n \rightarrow \infty$. In a similar manner, if $\delta-a_{2}<0$, then

$$
Q=\frac{a_{2}^{n+2 m}-\delta^{n+2 m}}{a_{2}-\delta} \leq \frac{a_{2}^{n+2 m}}{a_{2}-\delta}
$$

and this converge to 0 as $n \rightarrow \infty$. This reasoning is valid, since, from the theorem's assumptions, we know that $0 \leq a_{2}<1$ and $\delta<\frac{1}{s}<1$. So, in this case, since $Q \rightarrow 0$, then $d\left(x_{n}, x_{n+2 m}\right) \rightarrow 0$, as $n \rightarrow \infty$.

So, from both cases, we have shown that $\left(x_{n}\right)$ is a b-rectangular Cauchy sequence. Also, we know that $x_{n} \neq x_{m}$, for each $n \neq m$ and that $(X, d)$ is complete. This means that there exists $u \in X$, such that $\lim _{n \rightarrow \infty} x_{n}=u$.

Moreover, since the contractive condition can be reduced to the original form, i.e. $a d(x, f x)+b d(y, f y)+c d(f x, f y) \leq k d(x, y)$, then, as in the proof of (Theorem 2.1), there exists a unique point $u$ of $f$, as long as $a+c>0$ and $c<k$. 
Finally, we give an example regarding (Theorem 2.9).

Example 2.10. Let $(X, d)$, with $X=\{1,2,3,4\}$ be the b-rectangular metric space, endowed with the b-rectangular metric from (Example 2.2). Define a self-mapping $f$, by: $f(1)=2, f(2)=3, f(3)=1$ and $f(4)=4$. It is obviously that $f$ has as a unique fixed point the element $4 \in X$. We will determine the coefficients $\alpha, \beta$ and $\gamma$, such that $f$ satisfies $d(f x, f y) \geq \alpha d(x, y)+\beta d(x, f x)+\gamma d(y, f y)$ :

By $x=2$ and $y=1$, we get that $\frac{1}{10} \geq \alpha \frac{6}{10}+\beta \frac{1}{10}+\gamma \frac{6}{10}$

By $x=1$ and $y=2$, we get that $\frac{1}{10} \geq \alpha \frac{6}{10}+\beta \frac{6}{10}+\gamma \frac{1}{10}$

By $x=1$ and $y=3$, we get that $\frac{6}{10} \geq \alpha \frac{1}{10}+\beta \frac{6}{10}+\gamma \frac{1}{10}$

By $x=3$ and $y=1$, we get that $\frac{6}{10} \geq \alpha \frac{1}{10}+\beta \frac{1}{10}+\gamma \frac{6}{10}$

By $x=1$ and $y=4$, we get that $\frac{2}{10} \geq \alpha \frac{2}{10}+\beta \frac{6}{10}+\gamma \frac{2}{10}$

By $x=4$ and $y=1$, we get that $\frac{2}{10} \geq \alpha \frac{2}{10}+\beta \frac{2}{10}+\gamma \frac{6}{10}$

By $x=3$ and $y=2$, we get that $\frac{1}{10} \geq \alpha \frac{1}{10}+\beta \frac{1}{10}+\gamma \frac{1}{10}$

By $x=2$ and $y=3$, we get that $\frac{1}{10} \geq \alpha \frac{1}{10}+\beta \frac{1}{10}+\gamma \frac{1}{10}$

By $x=4$ and $y=2$, we get that $\frac{2}{10} \geq \alpha \frac{2}{10}+\beta \frac{2}{10}+\gamma \frac{1}{10}$

By $x=2$ and $y=4$, we get that $\frac{2}{10} \geq \alpha \frac{2}{10}+\beta \frac{1}{10}+\gamma \frac{2}{10}$

By $x=4$ and $y=3$, we get that $\frac{2}{10} \geq \alpha \frac{2}{10}+\beta \frac{2}{10}+\gamma \frac{1}{10}$

By $x=3$ and $y=4$, we get that $\frac{2}{10} \geq \alpha \frac{2}{10}+\beta \frac{1}{10}+\gamma \frac{2}{10}$

By $x=y$, we get that $\beta+\gamma \leq 0$

Now, we observe that (2.11) and (2.14) are equivalent relations. Also, we shall employ the more restrictive conditions on the coefficients $\alpha, \beta$ and $\gamma$, i.e. inequalities (2.11), (2.3), (2.5), (2.7), (2.8) and (2.14). Furthermore, we shall impose more restrictive conditions such that the number of inequalities is reduced: instead of (2.11) and (2.3), we impose that $1 \geq 6 \alpha+\beta+2 \gamma$, instead of (2.7) and (2.8) we require only (2.7) and instead of $1 \geq 6 \alpha+\beta+2 \gamma$ and (2.5), we require $1 \geq 6 \alpha+\beta+6 \gamma$. We mention that all of the above reasoning was made under the assumptions that $\beta \leq 0$ and $\gamma>0$.

Now, we have only two conditions, along with the conditions from (Theorem 2.9), when $\alpha>\gamma$

$$
\left\{\begin{array}{l}
\beta+\gamma \leq 0,1 \geq 6 \alpha+\beta+6 \gamma \\
\beta<1-s, \gamma>s, \alpha \gamma \\
\alpha+\gamma<\frac{1-\beta}{s}, \alpha+1<\gamma\left(1+\frac{1}{s}\right)
\end{array}\right.
$$


Now, taking account of the fact that $s=\frac{3}{2}$, we can find some values for the coefficients $\alpha, \beta$ and $\gamma$. For example, the inequalities are satisfied when $\alpha=\frac{9}{50}, \beta=-\frac{101}{5}$ and $\gamma=\frac{17}{100}$.

Now, we recall (Lemma 2) from [5], that is crucial for inequalities involving difference inequations.

Lemma 2.11. Let $\left(a_{n}\right)$ and $\left(b_{n}\right)$ be two sequences of nonnegative real numbers, such that

$$
a_{n+1} \leq \alpha_{1} a_{n}+\alpha_{2} a_{n-1}+\ldots+\alpha_{k} a_{n-k+1}+b_{n}, \text { where } n \geq k-1
$$

If $\alpha_{1}, \ldots, \alpha_{k} \in[0,1), \sum_{i=1}^{k} \alpha_{i}<1$ and $\lim _{n \rightarrow \infty} b_{n}=0$, then it follows that $\lim _{n \rightarrow \infty} a_{n}=0$.

Remark 2.12. In the previous proof, we have shown that the following estimation is valid

$$
d_{n}^{*}=d\left(x_{n+2}, x_{n}\right) \leq a_{2}^{n} d_{0}^{*}+\frac{\delta^{n}-a_{2}^{n}}{\delta-a_{2}} a_{1} D_{0} .
$$

So, based on this lemma, we give a nonconstructive approach for evaluating $\left(x_{n}\right)$ as a Cauchy sequence.

In the above lemma, let's take $k=1$. Then, we get that $a_{n+1} \leq \alpha_{1} a_{n}+b_{n}$, with $\alpha_{1} \in[0,1)$ and $\lim _{n \rightarrow \infty} b_{n}=0$. Then $\lim _{n \rightarrow \infty} a_{n}=0$.

Now, we have proved that $d_{n}^{*} \leq a_{2} d_{n-1}^{*}+a_{1} \delta^{n-1} D_{0}$.

Let's define the following: $\alpha_{1}:=a_{2}$ and $b_{n}:=a_{1} D_{0} \delta^{n-1}$. Since $\delta<\frac{1}{s}<1$ and $a_{2} \in[0,1)$, then apply (Lemma 2) from [5] with the particular case when $k=1$, we get that $\lim _{n \rightarrow \infty} d_{n}^{*}=0$.

Now, we give a proof for expansive-type mappings under the new assumption such that the mapping $f$ is onto and we shall use the 'inverse' Picard iterative process.

Theorem 2.13. Let $(X, d)$ be a complete b-rectangular metric space and $f: X \rightarrow X a$ mapping satisfying

$$
d(f x, f y) \geq \alpha d(x, y)+\beta d(x, f x)+\gamma d(y, f y)
$$

Let $f$ continuous and onto. Suppose that

(i) $\beta<1, \alpha+\gamma>0$ and $1-\beta<\frac{\alpha+\gamma}{s}$.

Also, suppose the following additional assumptions

Case (E1), i.e. $\alpha>0$ : Suppose that the following assumptions are satisfied:

(ii) $\alpha>1$

Case (E2), i.e. $\alpha<0$ : Suppose the following assumptions are satisfied:

(ii) $\alpha<-1, \gamma>0$

(iii) $s\left(1-\frac{\alpha}{\gamma}\right)<1+\frac{1}{\alpha}$

Then, the mapping $f$ has a fixed point in $X$. 
Proof. Here, we know that $f$ is continuous and onto. Let $x_{0}$ be an arbitrary point. As we have shown in the previous theorem, i.e. (Theorem 2.9), we reduce the contractive condition to

$$
d(f x, f y) \geq \alpha d(x, y)+\beta d(x, f x)+\gamma d(y, f y)
$$

Because $f$ is an onto mapping, by definition, we have that for each $y \in X$, there exists $x \in X$, such that $y=f x$.

Now, for $x_{0} \in X$, there exists $x_{1} \in X$, such that $x_{0}=f x_{1}$. Also, for $x_{1} \in X$, there exists $x_{2} \in X$, such that $x_{1}=f x_{2}$. Inductively, we get that $x_{n}=f x_{n+1}$, for each $n \in \mathbb{N}$.

Applying the contractive condition on the pair $\left(x_{n+1}, x_{n}\right)$, it follows that:

$$
\begin{aligned}
& d\left(f x_{n+1}, f x_{n}\right) \geq \alpha d\left(x_{n}, x_{n+1}\right)+\beta d\left(x_{n}, f x_{n}\right)+\gamma d\left(x_{n+1}, f x_{n+1}\right) \\
& d\left(x_{n}, x_{n-1}\right) \geq \alpha d\left(x_{n}, x_{n+1}\right)+\beta d\left(x_{n}, x_{n-1}\right)+\gamma d\left(x_{n+1}, x_{n}\right) \\
& \Longrightarrow(\alpha+\gamma) d\left(x_{n+1}, x_{n}\right) \leq(1-\beta) d\left(x_{n-1}, x_{n}\right) \\
& \Longrightarrow d\left(x_{n}, x_{n+1}\right) \leq \theta d\left(x_{n-1}, x_{n}\right),
\end{aligned}
$$

where $\theta:=\frac{1-\beta}{\alpha+\gamma}$. From the hypothesis, we know that $\theta \in\left[0, \frac{1}{s}\right)$, because $\beta<1$, $\alpha+\gamma>0$ and $1-\beta<\frac{\alpha+\gamma}{s}$. Furthermore, we have that $d_{n+1}:=d\left(x_{n+1}, x_{n}\right) \leq \theta^{n} d_{1}$. For simplicity, let's denote by $D_{0}:=d_{1}=d\left(x_{1}, x_{0}\right)$.

Furthermore, as in the previous theorem, let $d_{n}^{*}:=d\left(x_{n}, x_{n+2}\right)$, for each $n \in \mathbb{N}$.

Now, we shall analyze two different cases for estimation of $d\left(x_{n}, x_{n+2}\right)$

Case (E1): When $\alpha>0$, or with the original notation, $\frac{k}{c}>0$. Since $c<0$, we get that $k<0$.

Applying the expansive-type condition on the pair $\left(x_{n}, x_{n+2}\right)$, it follows that

$$
\begin{aligned}
& d\left(x_{n-1}, x_{n+1}\right)=d\left(f x_{n}, f x_{n+2}\right) \geq \alpha d\left(x_{n}, x_{n+2}\right)+\beta d\left(x_{n}, f x_{n}\right)+\gamma d\left(x_{n+2}, f x_{n+2}\right) \\
& =\alpha d\left(x_{n}, x_{n+2}\right)+\beta d\left(x_{n}, x_{n-1}\right)+\gamma d\left(x_{n+1}, x_{n+2}\right) \Longrightarrow \\
& \alpha d\left(x_{n}, x_{n+2}\right) \leq d\left(x_{n-1}, x_{n+1}\right)-\beta d\left(x_{n-1}, x_{n}\right)-\gamma d\left(x_{n+1}, x_{n+2}\right) \\
& d\left(x_{n}, x_{n+2}\right) \leq \frac{1}{\alpha} d_{n-1}^{*}+\left(-\frac{\beta}{\alpha}\right) d_{n}+\left(-\frac{\gamma}{\alpha}\right) d_{n+2} \\
& d\left(x_{n}, x_{n+2}\right) \leq \frac{1}{\alpha} d_{n-1}^{*}+\left(\left|\frac{\beta}{\alpha}\right|\right) d_{n}+\left(\left|\frac{\gamma}{\alpha}\right|\right) d_{n+2}
\end{aligned}
$$

Since $d_{n+1} \leq \theta^{n} D_{0}$, so $d_{n} \leq \theta^{n-1} D_{0}$, it follows that

$$
d_{n}^{*} \leq \frac{1}{\alpha} d_{n-1}^{*}+\theta^{n-1} Q D_{0}, \text { where } Q:=\left|\frac{\beta}{\alpha}\right|+\left|\frac{\gamma}{\alpha}\right| \theta^{3} .
$$

Since $\theta \in\left[0, \frac{1}{s}\right) \subset[0,1)$ and $\alpha>1$, we get, by (Lemma 2) in [5] and by (Lemma 2.11), that $\lim _{n \rightarrow \infty} d_{n}^{*}=0$. Now, as in the proof of (Theorem 2.9), we give a constructive approach for the upper bound of $d\left(x_{n}, x_{n+p}\right)$. Furthermore, we shall omit the details. We know that $d_{n}^{*} \leq a_{2} d_{n-1}^{*}+a_{1} \theta^{n-1} D_{0}$, briefly $d_{n}^{*} \leq a_{2}^{n} d_{0}^{*}+\frac{\theta^{n}-a_{2}^{n}}{\theta-a_{2}} a_{1} D_{0}$, where 
$a_{1}:=Q$ and $a_{2}:=\frac{1}{\alpha}$. When $p=2 m+1$, then $d\left(x_{n}, x_{n+2 m+1}\right) \leq \frac{1+\theta}{1-s \theta^{2}} s \theta^{n} D_{0}$, and, by hypothesis, $s \theta^{2}<1$, then $d\left(x_{n}, x_{n+2 m+1}\right)$ converges to 0 .

When $p=2 m$, then $d_{n+2 m}^{*} \leq a_{2}^{n+2 m} d_{0}^{*}+\frac{\theta^{n+2 m}-a_{2}^{n+2 m}}{\theta-a_{2}} a_{1} D_{0}$. Since $\theta<\frac{1}{s}<1$ and $a_{2}<1$, by theorem's assumptions, then $d_{n+2 m}^{*}$ converges to 0 .

Moreover, $d\left(x_{n}, x_{n+2 m}\right) \leq \frac{1+\theta}{1-s \theta^{2}} s \theta^{n} D_{0}+s^{m-1} d_{n+2 m}^{*}$.

Case (E2): When $\alpha<0$. We shall use (Lemma 2.7):

We know that $d\left(x_{n}, x_{n+1}\right) \leq \theta d\left(x_{n-1}, x_{n}\right)$, for each $n \geq 1$.

As in the previous case, with the remark that we divide by $\alpha<0$, we get that

$$
d\left(x_{n}, x_{n+2}\right) \geq A d_{n-1}^{*}+B d_{n}+C d_{n+1}, \text { where } A:=\frac{1}{\alpha}, B:=\frac{\beta}{|\alpha|} \text { and } C:=\frac{\gamma}{|\alpha|} \text {. }
$$

By (Lemma 2.7), we get that

$$
\begin{aligned}
& C d_{n+1} \geq \varphi(C) d_{n+1}^{*}+\psi(C) d_{n+3}+\psi(C) d_{n}^{*} \\
& d_{n}^{*} \geq A d_{n-1}^{*}+B d_{n}+\varphi(C) d_{n+1}^{*}+\psi(C) d_{n+3}+\psi(C) d_{n}^{*} \\
& \varphi(C) d_{n+1}^{*} \leq d_{n}^{*}[1-\psi(C)]+(-A) d_{n-1}^{*}-\varphi(C) d_{n+3}-B d_{n}
\end{aligned}
$$

Since, by theorem's assumptions, $\varphi(C)>0$, we get that

$$
\begin{aligned}
& d_{n+1}^{*} \leq \frac{1-\psi(C)}{\varphi(C)} d_{n}^{*}-A d_{n-1}^{*}-\left[\varphi(C) d_{n+3}+B d_{n}\right] \\
& d_{n+1}^{*} \leq \frac{1-\psi(C)}{\varphi(C)} d_{n}^{*}-A d_{n-1}^{*}+\left[|\varphi(C)| d_{n+3}+|B| d_{n}\right] \\
& d_{n+1}^{*} \leq \frac{1-\psi(C)}{\varphi(C)} d_{n}^{*}-A d_{n-1}^{*}+\left[|\varphi(C)| \theta^{2}+|B|\right] \theta^{n} D_{0}
\end{aligned}
$$

On the other hand, let's denote by $b_{n}:=\left[|\varphi(C)| \theta^{2}+|B|\right] \theta^{n} D_{0}, \alpha_{1}:=\frac{1-\psi(C)}{\varphi(C)}$ and by $\alpha_{2}:=-A$. Since $\gamma>0$ and $C=\frac{\gamma}{|\alpha|}>0$, then $\varphi(C)=\frac{C}{s}>0$. Also, from $C>0$, then $\psi(C)=-C<0$. Now, $\alpha_{1}>0$ requires that $-C<1$ and this is true since $C>0$. Moreover, $\alpha_{2}=-A=-\frac{1}{\alpha}>0$, because $\alpha<0$ and so $\frac{1}{\alpha}<0$. This means that $\alpha_{1}$ and $\alpha_{2}$ are positive, so the sum of these two is positive. Now, we want to validate if the sum of $\alpha_{1}$ and $\alpha_{2}$ is less than 1 .

$$
\alpha_{1}+\alpha_{2}=\frac{1-\psi(C)}{\varphi(C)}-A=\frac{1+C}{\frac{C}{s}}-\frac{1}{\alpha} .
$$

So $\alpha_{1}+\alpha_{2}<1$ is equivalent to $s\left(\frac{1+C}{C}\right)<1+\frac{1}{\alpha}$. Since $C=\frac{\gamma}{|\alpha|}=\frac{\gamma}{-\alpha}$, then $s\left(1-\frac{\alpha}{\gamma}\right)<1+\frac{1}{\alpha}$. Now, we have two sub-cases.

If $1-\frac{\alpha}{\gamma}<0$, then $\alpha-\gamma>0$, i.e. $\alpha>\gamma$, so this is false, because $\alpha<0$ and $\gamma>0$. 
So, the only valid case is when $1-\frac{\alpha}{\gamma}>0$, so $\alpha<\gamma$. Since $\alpha$ and $\gamma$ have different signs, this is also valid. Now, because $s\left(1-\frac{\alpha}{\gamma}\right)<1+\frac{1}{\alpha}$ and by the fact that the right hand side is positive, it follows that $1+\frac{1}{\alpha}>0$, i.e. $\alpha<-1$, which is valid by hypothesis assumptions. Since $\theta \in\left[0, \frac{1}{s}\right) \subset[0,1)$, then $\lim _{n \rightarrow \infty} b_{n}=0$.

Also, since $\alpha_{1}+\alpha_{2} \in[0,1), \alpha_{1} \in[0,1)$ and $\alpha_{2} \in[0,1)$, then $\lim _{n \rightarrow \infty} d_{n}^{*}=0$. The rest of the proof follows as usual.

Now, we give an example of a b-rectangular metric space, which is b-rectangular and validate (Theorem 2.13) through another example, showing that the hypotheses and conclusion of the already mentioned theorem are true also in b-metric spaces.

Example 2.14. Let $X=[0, \infty)$, endowed with $d: X \times X \rightarrow \mathbb{R}_{+}$, such that $d(x, y)=$ $(x-y)^{2}$, for each $x, y \in X$. Then $(X, d)$ is a complete b-metric space, with coefficient $s=2$. Then, it is also a complete b-rectangular metric space, with coefficient $s=4$.

Example 2.15. Let $X=[0, \infty)$, where $d$ is the above b-rectangular metric, with $s=4$. Define $f: X \rightarrow X$ as $f(x)=\frac{x+\delta_{1}}{\delta_{2}}$, with $\delta_{1}, \delta_{2} \geq 0$. It is easy to see that $f$ is continuous. Also, for each $y \in X$, there exists $x=y \delta_{2}-\delta_{1} \geq 0$, since $\delta_{1}$ and $\delta_{2}$ are positive, so $f$ is onto. Moreover:

$$
d(f x, f y)=(f x-f y)^{2}=\left|\frac{x+\delta_{1}}{\delta_{2}}-\frac{y+\delta_{1}}{\delta_{2}}\right|=\frac{1}{\delta_{2}}|x-y|^{2}=\frac{1}{\delta_{2}} d(x, y) .
$$

Let's take $\beta=0, \gamma=0$ and $\alpha=10$. Also, let $\delta<\frac{1}{s}$, i.e. $\delta_{2}<\frac{1}{4}$. For example: $\delta_{2}=\frac{1}{10}$ and $\delta_{1}=1$.

Then $f$ satisfies $d(f x, f y) \geq 10 d(x, y)$, for each $x, y \in X$.

As an open problem with respect to generalized contractions in b-rectangular metric spaces, we give the following.

Open Problem. Following [3], consider a self-mapping f defined on a complete brectangular space $(X, d)$ with coefficient $s \geq 1$, that satisfy

$$
a d(x, f x)+b d(y, f y)+c d(f x, f y)+e d(x, f y)+g d(y, f x) \leq k d(x, y) .
$$

Develop fixed point theorems for the self-mapping above, in the context of brectangular metric spaces, with suitable conditions on the coefficients $a, b, c, e, g, k$.

Acknowledgments. The author is grateful to the referees for their suggestions that contributed to the improvement of the paper.

\section{References}

[1] Aage, C.T., Salunke, J.N., Some fixed point theorems for expansion onto mappings on cone metric spaces, Acta Mathematica Sinica, English Series, 27(2011), no. 6, 1101-1106. 
[2] Alghamdi, M.A., Hussain, N., Salimi, P., Fixed point and coupled fixed point theorems on b-metric-like spaces, J. Ineq. Appl., 402(2013).

[3] Asadi, M., Some results of fixed point theorems in convex metric spaces, Nonlinear Func. Anal. Appl, 19(2014), no. 2, 171-175.

[4] Aydi, H., Karapinar, E., Moradi, S., Coincidence points for expansive mappings under c-distance in cone metric spaces, Int. Journal of Math. and Math. Sci., Vol. 2012, Art. ID 308921.

[5] Berinde, V., Păcurar, M., Stability of k-step fixed point iterative methods for some Presic type contractive mappings, J. Inequal. Appl., 149(2014).

[6] Branciari, A., A fixed point theorem of Banach-Caccioppoli type on a class of generalized metric spaces, Publ. Math. Debrecen, 57(2000), 31-37.

[7] Daheriya, R.D., Jain, R., Ughade, M., Some fixed point theorem for expansive type mapping in dislocated metric spaces, Internat. Sch. Research Network, ISRN Math. Analysis, Vol. 2012, Art. ID 376832, 5 pages.

[8] Ding, H.S., Ozturk, V., Radenovic, S., On some new fixed point results in b-rectangular metric spaces, J. Nonlinear Sci. Appl., 8(2015), 378-386.

[9] George, R., Radenovic, S., Reshma, K.P., Shukla, S., Rectangular b-metric space and contraction principles, J. Nonlinear Sci. Appl., 8(2015), 1005-1013.

[10] Kadelburg, Z., Radenovic, S., Fixed point results in generalized metric spaces without Hausdorff property, Math. Sci., 8(2014), 125.

[11] Kadelburg, Z., Radenovic, S., On generalized metric spaces: A survey, TWMS J. Pure Appl. Math., 5(2014), no. 1, 3-13.

[12] Kadelburg, Z., Radenovic, S., Pata-type common fixed point results in b-metric and b-rectangular metric spaces, J. Nonlinear Sci. Appl., 8(2015), 944-954.

[13] Karapinar, E., Fixed point theorems in cone Banach spaces, Fixed Point Theory Appl., Art. ID 609281 (2009).

[14] Kumar, S., Garg, S.K., Expansion mapping theorems in metric spaces, Int. J. Contemp. Math. Sciences, 4(2009), no. 36, 1749-1758.

[15] Moosaei, M., Fixed point theorems in convex metric spaces, Fixed Point Theory Appl., 164(2012).

[16] Moosaei, M., Common fixed points for some generalized contraction pairs in convex metric spaces, Fixed Point Theory Appl., 98(2014).

[17] Moosaei, M., Azizi, A., On coincidence points of generalized contractive pair mappings in convex metric spaces, J. of Hyperstructures, 4(2015), no. 2, 136-141.

[18] Olaoluwa, H., Olaleru, J.O., A hybrid class of expansive-contractive mappings in cone b-metric spaces, Afr. Mat.

[19] Pathak, H.K., Some fixed points of expansion mappings, Internat. J. Math. and Math. Sci., 19(1996), no. 1, 97-102.

[20] Patil, S.R., Salunke, J.N., Fixed point theorems for expansion mappings in cone rectangular metric spaces, Gen. Math. Notes, 29(2015), no. 1, 30-39.

[21] Rashwan, R.A,m Some fixed point theorems in cone rectangular metric spaces, Math. Aeterna, 2(2012), no. 6, 573-587.

[22] Roshan, J.R., Hussain, N., Parvaneh, V., Kadelburg, Z., New fixed point results in rectangular b-metric spaces, Nonlinear Analysis: Modelling and Control, 21(2016), 21, no. $5,614-634$. 
[23] Wang, C., Zhang, T., Approximating common fixed points for a pair of generalized nonlinear mappings in convex metric spaces, J. Nonlinear. Sci. Appl., 9(2016), 1-7.

[24] Zangenehmehr, P., Farajzadeh, A.P., Lashkaripour, P., Karamian, A., On fixed point theory for generalized contractions in cone rectangular metric spaces via scalarizing, Thai Journal of Math., 45(2012), no. 3, 717-724.

Cristian Daniel Alecsa

Babeş-Bolyai University

Faculty of Mathematics and Computer Sciences

Cluj-Napoca, Romania

e-mail: cristian.alecsa@math.ubbcluj.ro

"Tiberiu Popoviciu" Institute of Numerical Analysis

Romanian Academy

Cluj-Napoca, Romania

e-mail: cristian.alecsa@ictp.acad.ro 